Original paper

\title{
Impact of quantization algorithm and number of gray level intensities on variability and repeatability of low field strength magnetic resonance image-based radiomics texture features
}

\author{
Garrett Simpson, John C. Ford, Ricardo Llorente, Lorraine Portelance, Fei Yang, Eric A. Mellon, \\ Nesrin Dogan
}

Department of Radiation Oncology, University of Miami Miller School of Medicine, 1475 NW $12^{\text {th }}$ Ave, Miami, FL 33136, USA

\section{A R T I C L E I N F O}

\section{Keywords:}

Radiomics texture analysis

Quantization

Low field strength magnetic resonance image Preprocessing

\begin{abstract}
A B S T R A C T
Purpose: The purpose of this work was to investigate the impact of quantization preprocessing parameter selection on variability and repeatability of texture features derived from low field strength magnetic resonance (MR) images.

Methods: Texture features were extracted from low field strength images of a daily image QA phantom with four texture inserts. Feature variability over time was quantified using all combinations of three quantization algorithms and four different numbers of gray level intensities. In addition, texture features were extracted using the same combinations from the low field strength MR images of the gross tumor volume (GTV) and left kidney of patients with repeated set up scans. The impact of region of interest (ROI) preprocessing on repeatability was investigated with a test-retest study design.

Results: The phantom ROIs quantized to 64 Gy level intensities using the histogram equalization method resulted in the greatest number of features with the least variability. There was no clear method that resulted in the highest repeatability in the GTV or left kidney. However, eight texture features extracted from the GTV were repeatable regardless of ROI processing combination.

Conclusion: Low field strength MR images can provide a stable basis for texture analysis with ROIs quantized to 64 Gy levels using histogram equalization, but there is no clear optimal combination for repeatability.
\end{abstract}

\section{Introduction}

Radiomic texture analysis is the method of extracting statistical descriptors from oncologic images that quantify the frequency of spatial relationships of gray-level intensities [1]. These descriptors, called texture features, quantify the heterogeneity of intensities on a 2D or 3D level for use as diagnostic, predictive, or prognostic indicators [2-4]. Texture features capture patterns in images, both perceived and unrecognized by human observation [5]. The appeal of texture analysis stems from its ability to describe tumoral phenotypic heterogeneity quantitatively and non-invasively [6-14]. Many texture features are extracted from a region of interest (ROI) and mined for prognostic information, correlated with toxicities, or used as prognostic markers for treatment outcome [15-25]. With the advent of on-board magnetic resonance (MR)-guided radiotherapy machines, images acquired during routine treatment are under scrutiny for the applicability of radiomics texture analysis [26]. Quantitative analysis of patient set up images is also emerging as a route to evaluate disease microenvironment and phenotypic variation on an individual level [27].

Although radiomics texture analysis is capable of disease classification, patient stratification, and response prediction, there are many factors that impact feature values and may alter study results $[28,29]$. Factors that reduce the repeatability of texture features exist in every step ranging from image acquisition to feature extraction parameters. Lack of consistency in image acquisition parameters is a major barrier for translation of studies across multiple institutions [30]. The implementation of test-retest studies to determine which factors impact repeatability provides important guidance for future investigators. Utilization of daily images produced from a single machine may limit the impact of machine vendor, pulse sequence, image reconstruction, and field strength [31-37]. Studies have demonstrated MR images produced using different parameters and machines must be evaluated

\footnotetext{
* Corresponding author.

E-mail address: ndogan@med.miami.edu (N. Dogan).
} 
independently for not only the impact on texture feature values, but also the impact on their repeatability [33,36-41].

Image preprocessing is an important step prior to the extraction of texture features. Unlike other popular modalities, MR intensity values are not tissue specific. Computed tomography (CT) image intensities are density-linked (Hounsfield's Units) and positron emission tomography (PET) images are linked to radiotracer accumulation within the tissues. The MR image gray level intensities are not measurement-based as is in CT or PET and require processing to produce images on the same gray level scales [42]. Initially, the dynamic range of the images is limited using the Collewet method [43]. This method limits the original image's intensity values to \pm 3 standard deviations $(\sigma)$ of the mean intensity value $(\mu)$. It was demonstrated that limiting the range of initial intensity values was associated with superior classification of new and old cheese samples in low field strength (0.2 T) MR images. Following the Collewet method each MR image intensity range is transformed into a standardized range. The transformation of image intensities is especially relevant for texture analysis of MR images because multiple acquisitions of even the same subject can result in different of gray level intensity distributions. This transformation to a standard range is known as quantization and fixes the variable gray level ranges to a common intensity range for extraction of texture features. Different quantization methods transform the image using distinct algorithms that could assign intensity of the same voxel to a different discrete range of gray level value in the processed image. The selection of quantization method and the number of intensity levels may thus potentially impact texture feature stability or repeatability.

While MR imaging technology has shifted towards higher field strength magnets for diagnostic purposes, low field strength MR units are fulfilling a role as a daily set up imaging tool in radiotherapy settings and will continue to exist for the foreseeable future [44]. The impact of quantization and the number of bins (integer gray level intensities after quantization) on radiomics texture feature variability and repeatability of low field strength images remain unknown. Traverso et al. used Apparent Diffusion Coefficient (ADC) maps produced with $1.5 \mathrm{~T}$ and 3.0 T MR units to test texture feature sensitivity to five different normalization techniques and two quantization types (fixed number of bins and fixed bin width) [45]. Brynolfsson et al. extracted texture features from ADC maps with variation of the quantization method and the number of gray levels to observe the behavior of the texture features [46]. However, neither study used low field strength MR images or explored if selection of quantization method and number of gray level intensities impacted texture feature variation or repeatability. The purpose of this work is to investigate whether different image quantization methods and the number of gray level intensities impact texture feature variation in a texture phantom. Additionally, a test-retest analysis has been conducted by leveraging repeated clinical set up images to investigate the impact of the combination of quantization method and number of bin levels on feature repeatability.

\section{Materials and methods}

\subsection{Image data}

Two sets of images were utilized for this study. All images were acquired with two torso coils with six phased array elements with one coil placed posterior and one anterior. All images were acquired on the same $0.35 \mathrm{~T}$ split bore MR machine. The pulse sequenced used for acquisition is a balanced steady-state free procession pulse sequence (bSSFP) and is a coherent gradient echo sequence that utilizes fully balanced gradient waveforms. The first series of images included in the study was of a texture phantom and included a total of 37 images spanning 44 days. Each phantom image included was acquired with voxel dimensions of $1.5 \times 1.5 \times 1.5 \mathrm{~mm}^{3}$. The second set of images used for texture analysis were clinical patient set up images. The patient library consisted of 18 pairs of set up images for a total of 36 images of 11 institutional review board (IRB) approved protocol patients. Set up image pairs were all acquired within $30 \mathrm{~min}$. All patient images were acquired in the headfirst supine treatment position with voxel dimensions of $1.5 \times 1.5 \times$ $3.0 \mathrm{~mm}^{3}$. All imaging parameters and voxel dimensions are displayed in Table 1. Though patient images consisted of three minor variations of the same clinical pulse sequence, impact due to differences is minimized for two reasons: voxel dimensions were identical across all sequences, and TR and TE values were very similar having minimal impact to texture feature values [37]. All test-retest image pairs were acquired using the identical pulse parameters.

\subsection{Texture phantom}

A daily MR QA phantom (ViewRay, Inc.) was used as the base for four texture inserts. Four $6 \mathrm{ml}$ test tubes were filled with different materials to produce distinct image textures. The first tube was filled with only vitamin E pills. The second tube was filled with chopped up plastic intravenous (IV) tubing and brimmed with copper sulfate-doped $\left(\mathrm{CuSO}_{4}\right.$-doped) tap water. The third texture insert tube was filled with rolled-up medical $4 \mathrm{x} 4$ gauze sponge and brimmed with $\mathrm{CuSO}_{4}$-doped tap water. The final insert was constructed by filling the test tube with 6 $\mathrm{cm}$ long capillary tubes (micro-hematocrit tubes) and then filled with $\mathrm{CuSO}_{4}$-doped water. $\mathrm{CuSO}_{4}$-doped water was included to reduce the T1 relaxation time of the liquid portion within each tube. The texture inserts fit into holes in the phantom at the four cardinal angles approximately $5 \mathrm{~cm}$ from isocenter. The phantom rig is designed for image quality QA and ensures the position of the phantom and receiver coils are positioned nearly identically for each acquisition. See Fig. 1 for axial and sagittal views of each texture insert.

\subsection{ROI delineation}

Four regions of interest, the inside of each test tube, were defined on the first image in the series for texture feature extraction. ROI was contoured to include as much of the material designed to provide texture and exclude the plastic material of the tube along with any liquid without texture. The ROI for the tube filled with vitamin E pills will be referred to as ROI_E, the IV tubing as ROI_IV, the ROI containing the gauze as ROI_G, and the capillary tube volume as ROI_Cap. The contours were then rigidly transferred to the following daily images to ensure each ROI captured the identical volumes within each daily image.

Clinical image data was drawn from patients treated for pancreatic or liver lesions. Two structures were selected as ROIs for texture analysis, the left kidney and the gross tumor volume (GTV). The left kidney was selected because of the relative ease of contouring and presence in all image series. The GTV was selected because the tumor volume is often the ROI in texture analysis studies. A physician with extensive

\section{Table 1}

Display of pulse sequence parameters for all bSSFP images included for texture analysis. All images of the texture phantom were acquired using the same pulse parameters and voxel sizes. The patient image database consisted of three pulse sequences with identical voxel dimensions and flip angles.

\begin{tabular}{|c|c|c|c|c|}
\hline \multirow[t]{3}{*}{ Acquisition Parameter } & \multicolumn{4}{|c|}{ Pulse Sequences } \\
\hline & \multirow{2}{*}{$\begin{array}{l}\text { Phantom } \\
\text { Images }\end{array}$} & \multicolumn{3}{|c|}{ Patient Images } \\
\hline & & A & B & $\mathrm{C}$ \\
\hline TR/TE (ms) & $3.84 / 1.92$ & $3.00 / 1.27$ & $3.33 / 1.43$ & $3.36 / 1.44$ \\
\hline Flip angle $\left({ }^{\circ}\right)$ & 60 & 60 & 60 & 60 \\
\hline Bandwidth (Hz/pixel) & 532 & 604 & 537 & 534 \\
\hline Field of view (mm) & $\begin{array}{l}216 \times 268 \\
\times 280\end{array}$ & $\begin{array}{l}540 \times 465 \\
\times 432\end{array}$ & $\begin{array}{l}400 \times 400 \\
\times 432\end{array}$ & $\begin{array}{l}500 \times 450 \\
\times 432\end{array}$ \\
\hline Matrix size & $\begin{array}{l}144 \times 180 \\
\times 188\end{array}$ & $\begin{array}{l}360 \times 310 \\
\times 144\end{array}$ & $\begin{array}{l}266 \times 266 \\
\times 144\end{array}$ & $\begin{array}{l}334 \times 300 \\
\times 144\end{array}$ \\
\hline $\begin{array}{l}\text { Voxel dimensions } \\
\qquad(\mathrm{mm} \times \mathrm{mm} \times \mathrm{mm})\end{array}$ & $\begin{array}{l}1.5 \times 1.5 \times \\
1.5\end{array}$ & $\begin{array}{l}1.5 \times 1.5 \\
\times 3.0\end{array}$ & $\begin{array}{l}1.5 \times 1.5 \\
\times 3.0\end{array}$ & $\begin{array}{l}1.5 \times 1.5 \\
\times 3.0\end{array}$ \\
\hline Number of images & 36 & 6 (pairs) & 10 (pairs) & 2 (pairs) \\
\hline
\end{tabular}




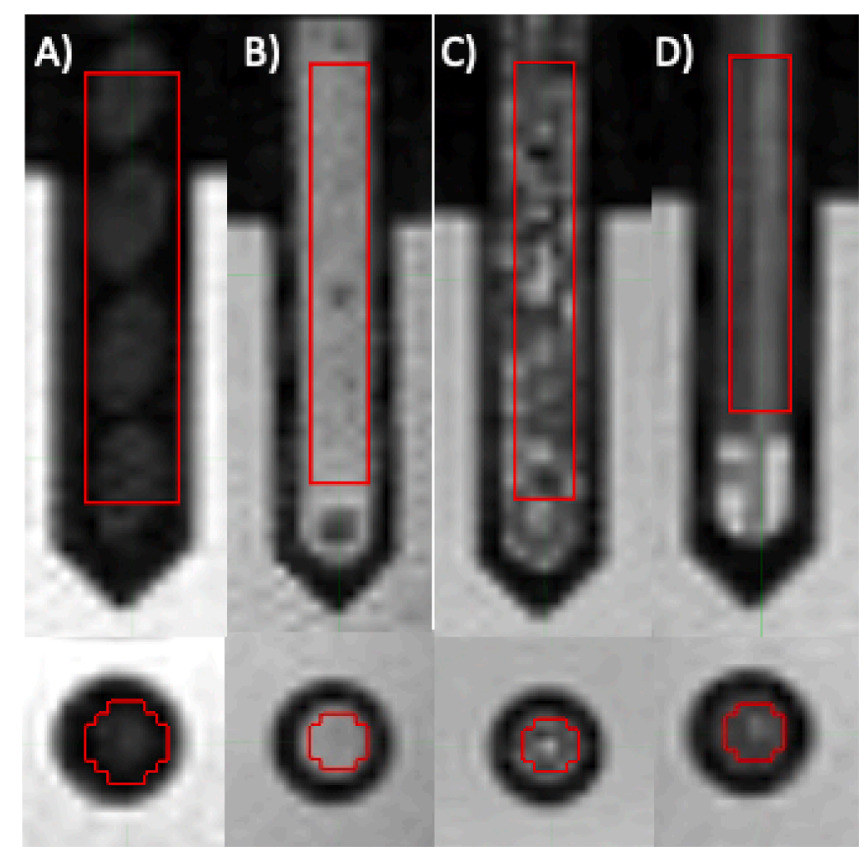

Fig. 1. Images of the four different texture inserts of the daily image QA phantom. Sagittal (top images) and axial (bottom images) slices of the four texture inserts with the ROI (red) defining the volume used to extract texture features. Column A is the vitamin E-pills (ROIE), column B is the test tube stuffed with gauze (ROI_G), column C is cut sections of IV tubing (ROI_IV), and column D is the tube filled with capillary tubes (ROI_Cap). (For interpretation of the references to colour in this figure legend, the reader is referred to the web version of this article.) gastrointestinal experience reviewed all left kidney contours and delineated all GTVs. Contouring was performed on the first image series (test images) and the resulting contours were then rigidly transferred to the second image series (retest images) after rigid alignment. An example of a test-retest pair of images is displayed, along with ROIs, in Fig. 2.

\subsection{Image quantization}

Prior to quantization, the dynamic range of the intensities was limited using the " $+/-3 \sigma$ " Collewet normalization method [43]. The quantization methods under consideration are Lloyd-Max quantization, uniform quantization, and equal-probability quantization method $[47,48]$. The three quantization methods under consideration are included in the publicly available Texture Analysis Toolbox in MATLAB (The MathWorks, Natick, Massachusetts) by Vallières et al. [49]. The input for all quantization algorithms was the dynamic range limited gray levels of the ROI. Each algorithm determines the corresponding gray level intensity value to assign the original intensity values to. The LloydMax algorithm iteratively minimizes the mean squared error between the ROI intensity values and the user-determined gray level intensity bins. Uniform quantization scales the ROI intensity range linearly to the gray level intensity range and rounds to the nearest integer value. Equal probability quantization, also called histogram equalization, re-assigns ROI intensity values to bins such that the probability of each bin occurring are approximately equal. The histograms of both the LloydMax and Uniform methods result in a close histogram approximation of the original data's histogram. The equal probability method produces a flattened histogram after quantization and results in increased contrast across the image. Using all three methods, each ROI was re-quantized to $32,64,128$, and 256 intensity levels prior to texture analysis.
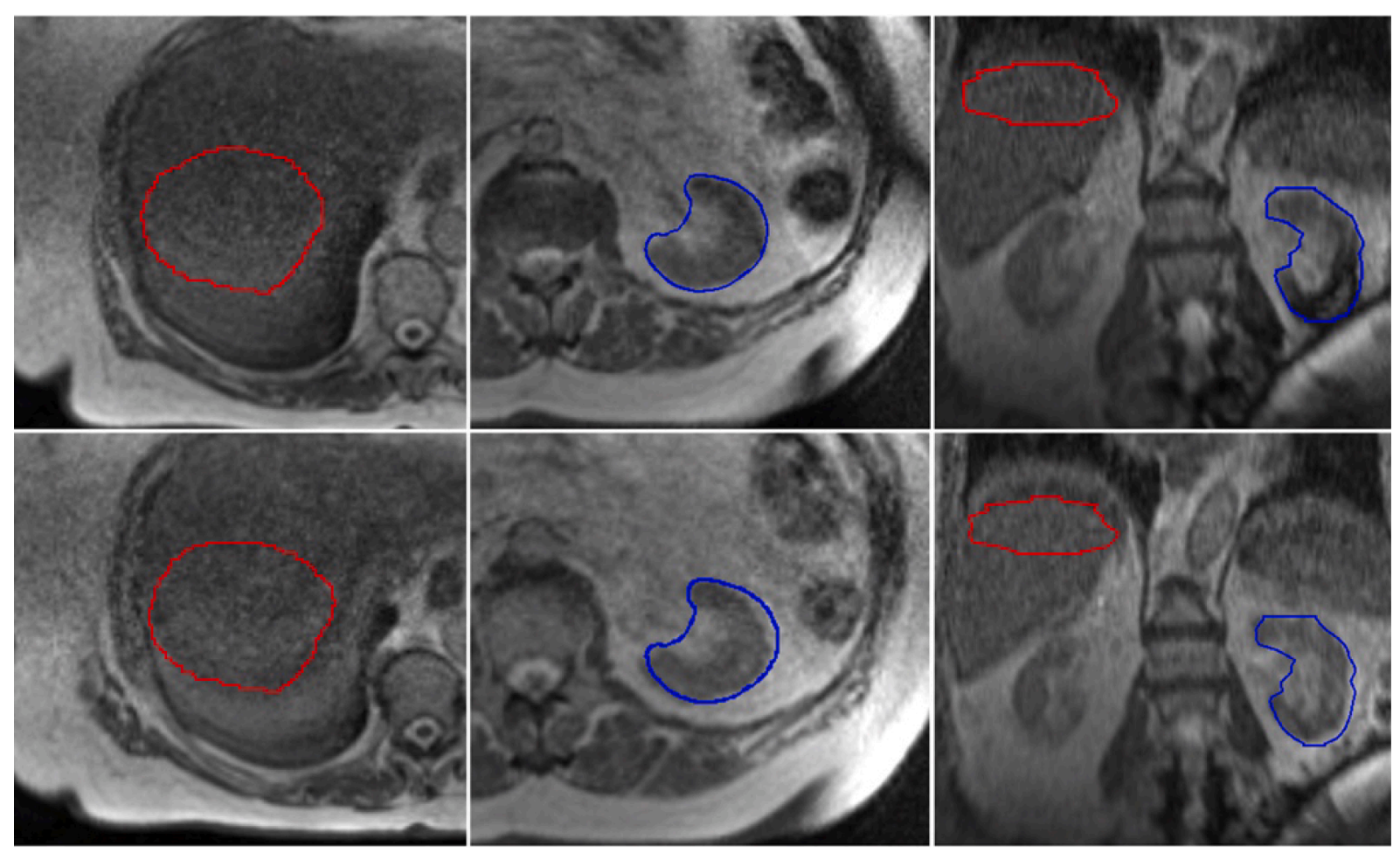

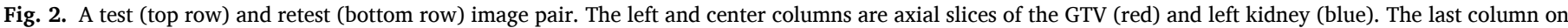

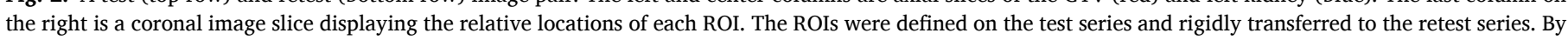

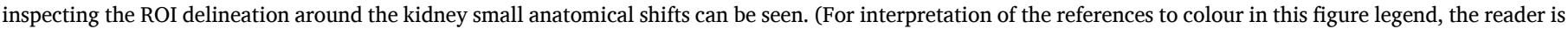
referred to the web version of this article.) 


\subsection{Texture analysis}

From each ROI all twelve combinations of method and number of bins were extracted from the initial image and the transferred delineations on the rescanned images. All features were calculated on a 3D basis. A total of 39 texture features belonging to gray-level co-occurrence matrix (GLCM), neighborhood gray tone difference matrix (NGTDM), gray-level size zone matrix (GLSZM), and gray-level run

\section{Table 2}

Texture features under investigation for stability and repeatability. The left column is the feature classes (encoding method), the next column is the IBSI code and aggregation method code. The third column contains the included texture features with abbreviations in the parenthesis. The column on the right has the corresponding IBSI feature codes. Texture features with * were calculated as defined by Amadasun and King [53].

\begin{tabular}{|c|c|c|c|}
\hline $\begin{array}{l}\text { Encoding } \\
\text { Method }\end{array}$ & $\begin{array}{l}\text { IBSI code/ } \\
\text { aggregation code }\end{array}$ & Texture feature: & $\begin{array}{l}\text { IBSI } \\
\text { Name }\end{array}$ \\
\hline \multirow[t]{8}{*}{ GLCM } & \multirow[t]{8}{*}{ LFYI/IAZD } & Energy & 8ZQL \\
\hline & & Contrast & ACUI \\
\hline & & Entropy & TU9B \\
\hline & & Homogeneity & IB1Z \\
\hline & & Correlation & NI2N \\
\hline & & Sum Average & ZGXS \\
\hline & & Variance & UR99 \\
\hline & & Dissimilarity & $8 S 9 J$ \\
\hline \multirow[t]{13}{*}{ GLRLM } & \multirow[t]{13}{*}{ TPOI/IAZD } & Short run emphasis (SRE) & $220 \mathrm{~V}$ \\
\hline & & Long run emphasis (LRE) & W4KF \\
\hline & & $\begin{array}{l}\text { Gray-level nonuniformity } \\
\text { (GNU) }\end{array}$ & R5YN \\
\hline & & $\begin{array}{l}\text { Run length nonuniformity } \\
\text { (RLN) }\end{array}$ & W92Y \\
\hline & & Run percentage (RP) & 9ZK5 \\
\hline & & $\begin{array}{l}\text { Low gray-level run emphasis } \\
\text { (LGRE) }\end{array}$ & V3SW \\
\hline & & $\begin{array}{l}\text { High gray-level run emphasis } \\
\text { (HGRE) }\end{array}$ & G3QZ \\
\hline & & $\begin{array}{l}\text { Short run low gray-level } \\
\text { emphasis (SRLGE) }\end{array}$ & HTZT \\
\hline & & $\begin{array}{l}\text { Short run high gray-level } \\
\text { emphasis (SRHGE) }\end{array}$ & GD3A \\
\hline & & $\begin{array}{l}\text { Long run low gray-level } \\
\text { emphasis (LRLGE) }\end{array}$ & IVPO \\
\hline & & $\begin{array}{l}\text { Long run high gray-level } \\
\text { emphasis (LRHGE) }\end{array}$ & 3KUM \\
\hline & & Gray-level variance (GLV) & 8CE5 \\
\hline & & Run length variance (RLV) & SXLW \\
\hline \multirow[t]{13}{*}{ GLSZM } & \multirow[t]{13}{*}{ 9SAK/КOBO } & Small zone emphasis (SZE) & 5QRC \\
\hline & & Large zone emphasis (LZE) & 48P8 \\
\hline & & $\begin{array}{l}\text { Gray-level nonuniformity } \\
\text { (GNU) }\end{array}$ & BYLV \\
\hline & & $\begin{array}{l}\text { Zone-size nonuniformity } \\
\text { (ZSN) }\end{array}$ & $4 J P 3$ \\
\hline & & Zone percentage (ZP) & P30P \\
\hline & & $\begin{array}{l}\text { Low gray-level zone emphasis } \\
\text { (LGZE) }\end{array}$ & XMSY \\
\hline & & $\begin{array}{l}\text { High gray-level zone emphasis } \\
\text { (HGZE) }\end{array}$ & $5 \mathrm{GN} 9$ \\
\hline & & $\begin{array}{l}\text { Small zone low gray-level } \\
\text { emphasis (SZLGE) }\end{array}$ & 5RAI \\
\hline & & $\begin{array}{l}\text { Small zone high gray-level } \\
\text { emphasis (SZHGE) }\end{array}$ & HW1V \\
\hline & & $\begin{array}{l}\text { Large zone low gray-level } \\
\text { emphasis (LZLGE) }\end{array}$ & YH51 \\
\hline & & $\begin{array}{l}\text { Large zone high gray-level } \\
\text { emphasis (LZHGE) }\end{array}$ & $\mathrm{J} 17 \mathrm{~V}$ \\
\hline & & Gray-level variance (GLV) & BYLV \\
\hline & & Zone-size variance (ZSV) & 3NSA \\
\hline \multirow[t]{5}{*}{ NGTDM } & \multirow[t]{5}{*}{ IPET/KOBO } & Coarseness* & - \\
\hline & & Contrast & $65 \mathrm{HE}$ \\
\hline & & Busyness & NQ30 \\
\hline & & Complexity & HDEZ \\
\hline & & Strength* & - \\
\hline
\end{tabular}

length matrix (GLRLM) classes were considered in this work, see Table 2 [50-57]. Features were aggregated using methods defined in the Image Biomarker Standardization Initiative (IBSI) [58]. The GLCM and GLRLM were aggregated from the 3D volume to form a single matrix (IBSI code: IAZD). The GLSZM and NGTDM were aggregated using 3D analysis (IBSI code: КОВO).

The GLCM-based features encode for the frequency of gray level spatial relationships, with the matrix coordinates representing the probability of two gray levels occurring as neighboring pair. GLNDMbased features consider the difference between each gray-level and the sum of the neighborhood (26-nearest neighbor voxels) around the voxel of interest. The number of occurrences of each gray-level is also recorded. GLSZM features quantify the size of isointense regions within the ROI. The matrix is populated by recording the probability of a region size of a single gray-level intensity occurring. The final class of texture features included for analysis are GLRLM. Where the GLSZM encodes the matrix with zone sizes of isointense regions, GLRLM encodes for connected runs of isointense voxels. Each texture feature, regardless of class, are calculated using a weighted sum over the entire matrix. Texture features emphasize uniuqe aspects of their respective class.

\subsection{Statistical analysis}

The variability of features extracted from the texture phantom using each combination of quantization method and number of bin levels was quantified using the coefficient of variation expressed as a percent (CV $\%$ ) and is simply the ratio of the standard deviation and the mean value. A CV\% below $10 \%$ is generally regarded as small variations and therefore stable [59]. The CV\% was calculated for 39 features preprocessed using each of the twelve calculation methods. The combination of quantization method and number of gray level intensity bins that produces the largest number of features stable across the four inserts was tested for sensitivity to phantom insert type. Each feature's sensitivity between the four inserts was tested using a one-way analysis of variance (ANOVA) to determine which texture features retain differentiation ability [60]. Furthermore, in order to test whether the phantoms' texture was representative of that in tumor, we calculated the Spearman rank correlation coefficient (SCC) between the 39 texture features in a GTV and one phantom image.

Next, the repeatability of each different combination of quantization method and the number of intensity levels was investigated using Lin's CCC [61]. The test data are the texture features extracted from the scan chronologically acquired first. The retest data are the features extracted from the rigidly transferred contours on the following set up scan. The repeatability of all methods and the number of bins was evaluated with Lin's CCC. The CCC was calculated with the upper and lower bounds of the $95 \%$ confidence interval and the corrections by McBride [62]. A CCC $\geq 0.85$ is a popular threshold for repeatability in texture analysis studies [31,63-65]. For this study a CCC $>0.80$ is selected as the threshold for consideration as being acceptably repeatable. Finally, the dependence of the repeatable texture features on GTV volume was examined by calculating the SCC of features in test image series and the retest image series. Features with an $|\mathrm{SCC}|>0.95$ in both datasets were considered volume dependent.

\section{Results}

The images of the texture inserts can be visually differentiated and characterized. ROI_E is easily characterized as relatively large volumes of higher signal intensities, inside the pills, surrounded by lower gray level intensities produced by air. The ROI_IV is constructed of many volumes of low signal intensity, the walls of the IV tube, and high signal, the $\mathrm{CuSO}_{4}$-doped water filling in around the plastic. ROI_G is characterized as an image constructed with higher gray level intensity signals as a result of the gauze being saturated with the water and providing high signal intensity across the entire volume. The ROI_Cap image is 
constructed of linearly structured regions of higher gray level intensities, the $\mathrm{CuSO}_{4}$-doped water filling in and around the capillary tubes, and regions of low signal in linear structures where the $\mathrm{CuSO}_{4}$-doped tap water is displaced by the walls of the capillary tubes.

The CV\% for each feature calculated with the various combinations of quantization method and number of bins are displayed in Figs. 3-6. Fig. 3 contains the results for ROI_E, Fig. 4 is ROI_IV, Fig. 5 is ROI_G, and Fig. 6 is the CV\% from features extracted from ROI_Cap. Each y-axis is labeled with the texture features, and the $\mathrm{x}$-axis is labeled with the quantization method and number of bins used when texture features were extracted for CV\% calculation. View across a single row compares a unique feature's CV\% across the calculation methods. Viewing a single column displays the $\mathrm{CV} \%$ of each texture feature for a single calculation method. GLCM-based entropy and homogeneity achieved a CV\% below $10 \%$ across all combinations of quantization method and number of bins for all phantom ROIs. GLRLM-based short run emphasis, long run emphasis, run-length non-uniformity, and run percentage had CV\% below $10 \%$ across all methods and texture inserts as well. No GLSZM or NGTDM-based features achieved a CV\% below 10\% across all methods and calculation combinations. Quantizing the ROIs with histogram equalization to $64 \mathrm{~Gy}$ levels provided the most texture features with low variability across all texture phantom inserts. Histogram equalization and 32 bin levels also performed well, resulting in many texture features with $\mathrm{CV} \%$ below $10 \%$. All texture features that were calculated from the texture ROIs processed using histogram equalization to 64 Gy level intensities were tested for sensitivity using the ANOVA test. By sensitivity we mean the ability of the texture feature to discriminate among the four phantoms. For all texture features, there was at least one texture insert that produced a statistically significant difference, $p$-value $<0.05$, in texture feature value. No post hoc tests were performed after the ANOVA test. Fig. 7 displays seven texture features determined to be stable using the $\mathrm{CV} \%$ threshold that were representative of all the stable features.

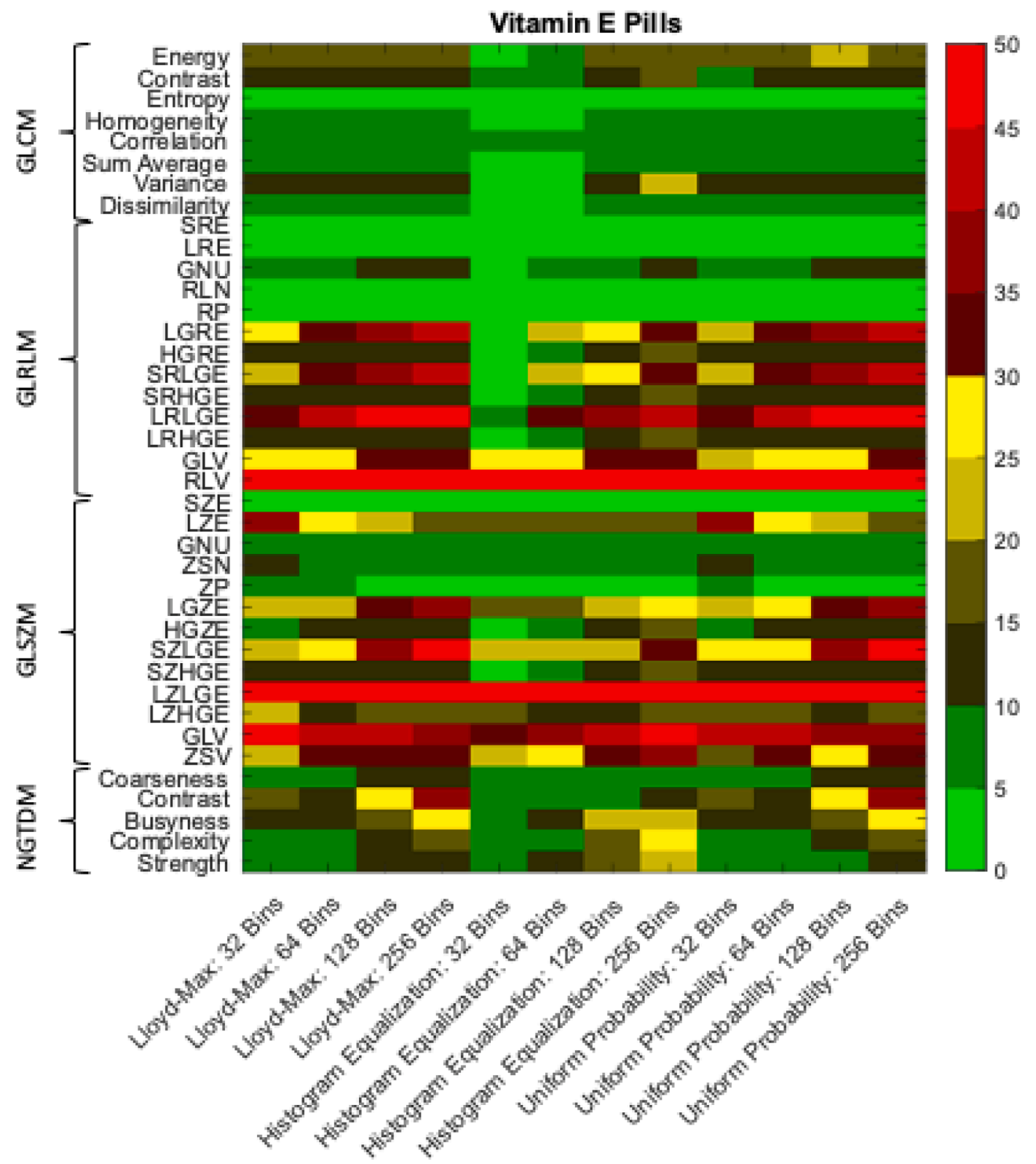

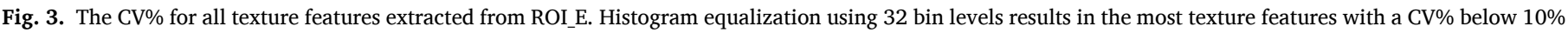

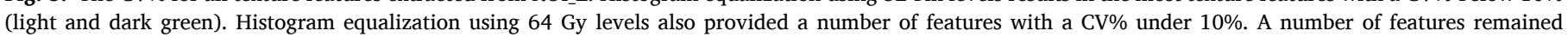

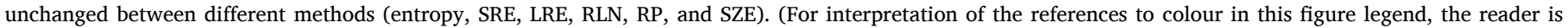
referred to the web version of this article.) 

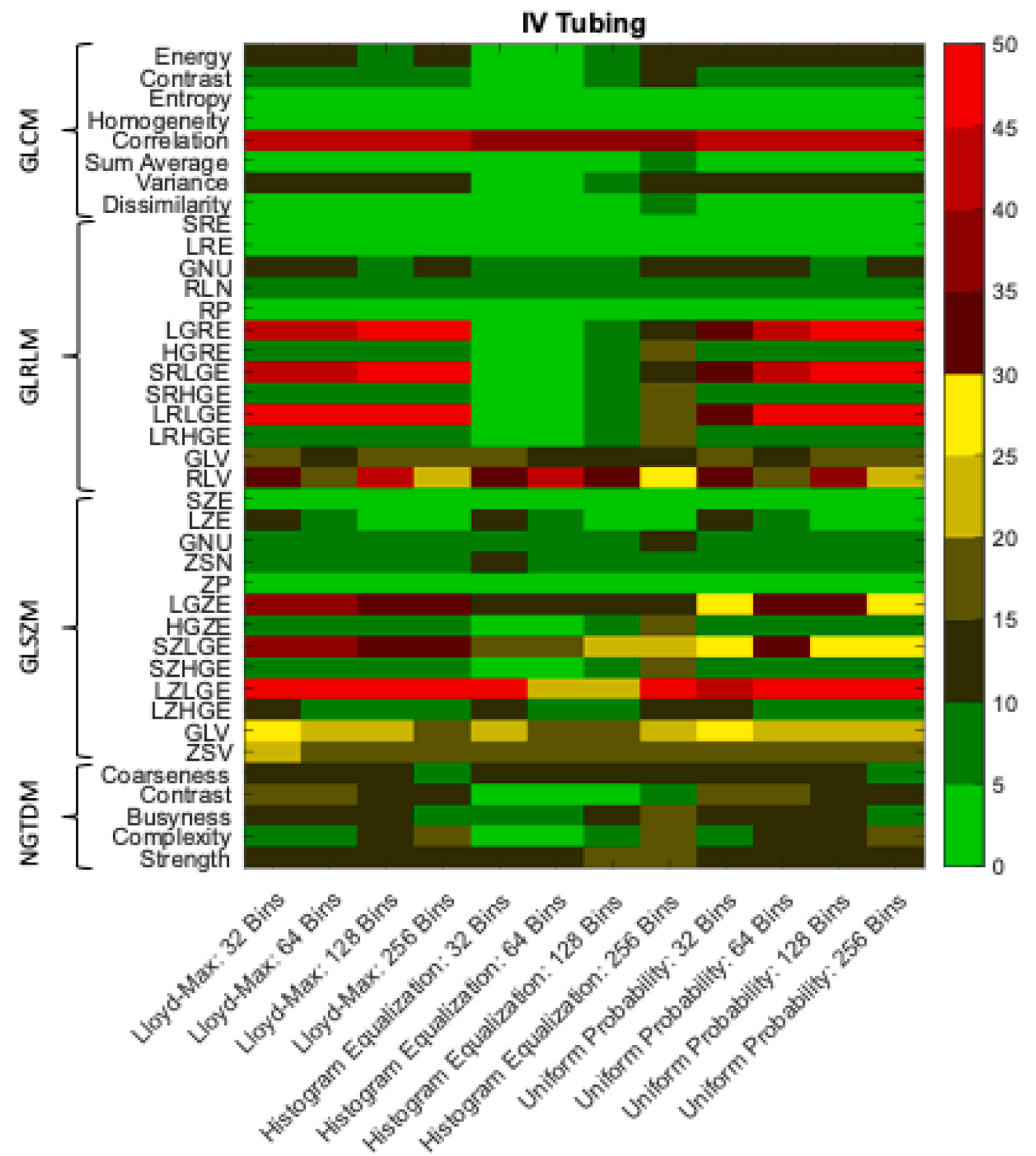

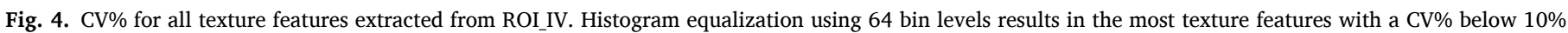

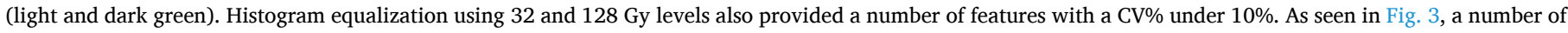

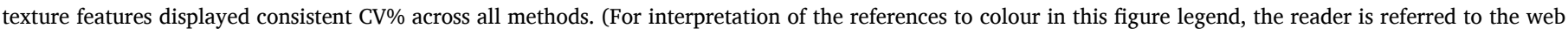
version of this article.)

The texture features extracted from the GTV were highly correlated with the phantom-based features calculated with histogram equalization to 32 Gy levels with $|\mathrm{SCC}|>0.94$ and $|\mathrm{SCC}|>0.95$ using histogram equalization and 64 levels. The correlation was calculated using histogram equalization to 32 and 64 Gy levels because of the low CV\% of each method.

Using a cutoff value of CCC $=0.80$ for features calculated from the GTV using the twelve combinations of quantization methods and graylevel intensity bin levels resulted in many similar features. There were eight texture features with a CCC $\geq 0.80$ across all combinations (see Table 3). Two features belong to GLRLM, three to GLSZM, and three to NGTDM. The number of features exceeding a CCC $=0.80$ for each combination varied slightly. Histogram equalization with the four levels resulted in 9, 9, 10, and 10 texture features for 32, 64, 128, and 256 levels, respectively. Uniform Probability resulted in 11, 12, 10, and 10 features, respectively. The Lloyd-Max algorithm resulted in 10, 11, 9, and 9 texture features for the respective number of levels. The test-retest analysis using the texture features calculated from the left kidney structure resulted in only five texture features with a CCC $\geq 0.80$ across all methods (see Table 4), two features belonging to GLRLM, one GLSZM-based feature, and two NGTDM-based features. In terms of the number of features exceeding the CCC cutoff, the number of features calculated using Histogram Equalization quantization was 9 for 32 intensity levels, 8 for 64,7 for 128 , and 6 for 256 intensity levels. The Uniform Probability quantization method resulted in 9 texture features for 32 and 64 intensity levels and 7 texture features for 64 and 128 intensity levels. Lloyd-Max algorithm resulted in 10, 8, 7, and 8 texture features for 32, 64, 128, and 256 intensity levels, respectively. Of the 9 repeatable features produced using histogram equalization and $32 \mathrm{~Gy}$ levels, GRLRM-based GLN and RLN were found to exceed the threshold of $|\mathrm{SCC}|=0.95$ in both the test and retest image series $(p<0.05)$. For the texture features extracted from GTVs processed using histogram 

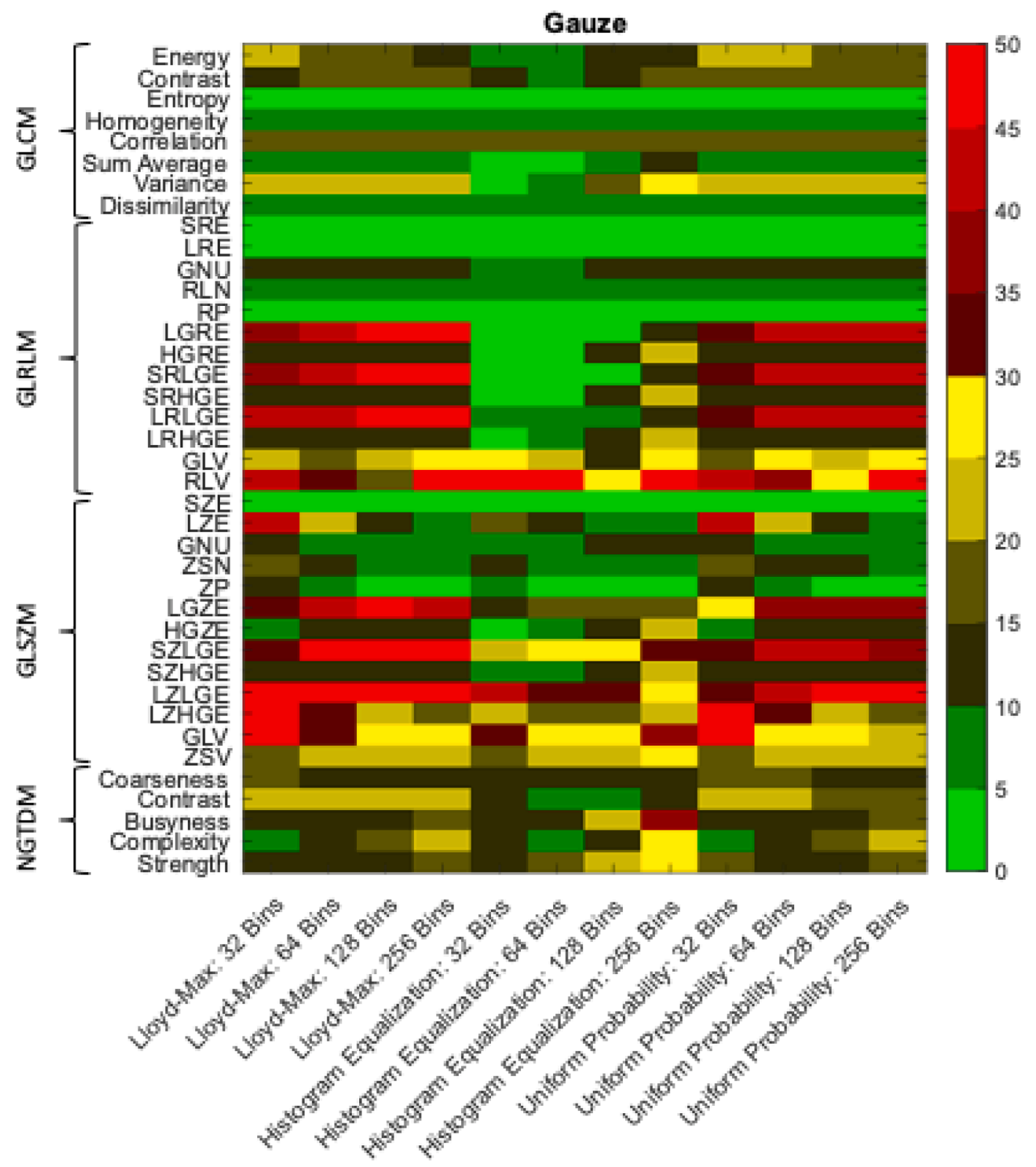

Fig. 5. CV\% for all texture features extracted from ROI_G. Histogram equalization using 64 bin levels results in the most texture features with a CV\% below $10 \%$ (light and dark green). Histogram equalization using 32 Gy levels also provided a number of features with a CV\% under 10\%. Entropy, homogeneity, dissimilarity, SRE, LRE, RLN, RP, and SZE maintained CV\% $<10 \%$. (For interpretation of the references to colour in this figure legend, the reader is referred to the web version of this article.)

equalization and 64 Gy levels, only GRLRM-based RLN exceeded the threshold for both image series $(p<0.05)$.

\section{Discussion}

The desire for higher quality diagnostic MR images has driven research to develop higher field strength magnets, but for the purpose of MR-guidance lower field strength magnets produce images with adequate contrast and resolution. With the introduction of MR-guided linacs, these images will remain clinically relevant. This study quantified the variation of texture features calculated using three different quantization methods and various gray-level intensities using a texture phantom with four synthetic texture inserts. This work aimed to explore the impact of choices in quantization algorithm and the number of bins on the stability and repeatability of texture features in low field strength MR images. Admittedly, combinations of quantization algorithm and the number of intensity bin levels that result in low variability and a higher degree of repeatability may not be ideal for capturing underlying biological heterogeneity. Requantization of images using too few graylevels could result in images that are similar due to limited dynamic range and texture features that are repeatable but useless because the gray-level heterogeneity has been smoothed over by preprocessing. The number of gray-levels is important for texture analysis because the number of levels determines the size of the encoding matrixes. Too many levels may result in a sparse matrix and too few levels may result in too dense of a matrix. Garpebring et al. highlight the importance of selecting the optimal number of gray-levels for calculation of texture features by demonstrating that some GLCM-based features can attain a minimum classification error by altering the GLCM size [66].

The CV\% calculation shows that using the Histogram Equalization quantization method with either 32 or 64 Gy-level bins results in lower variation across the texture features. Quantizing data using Histogram 


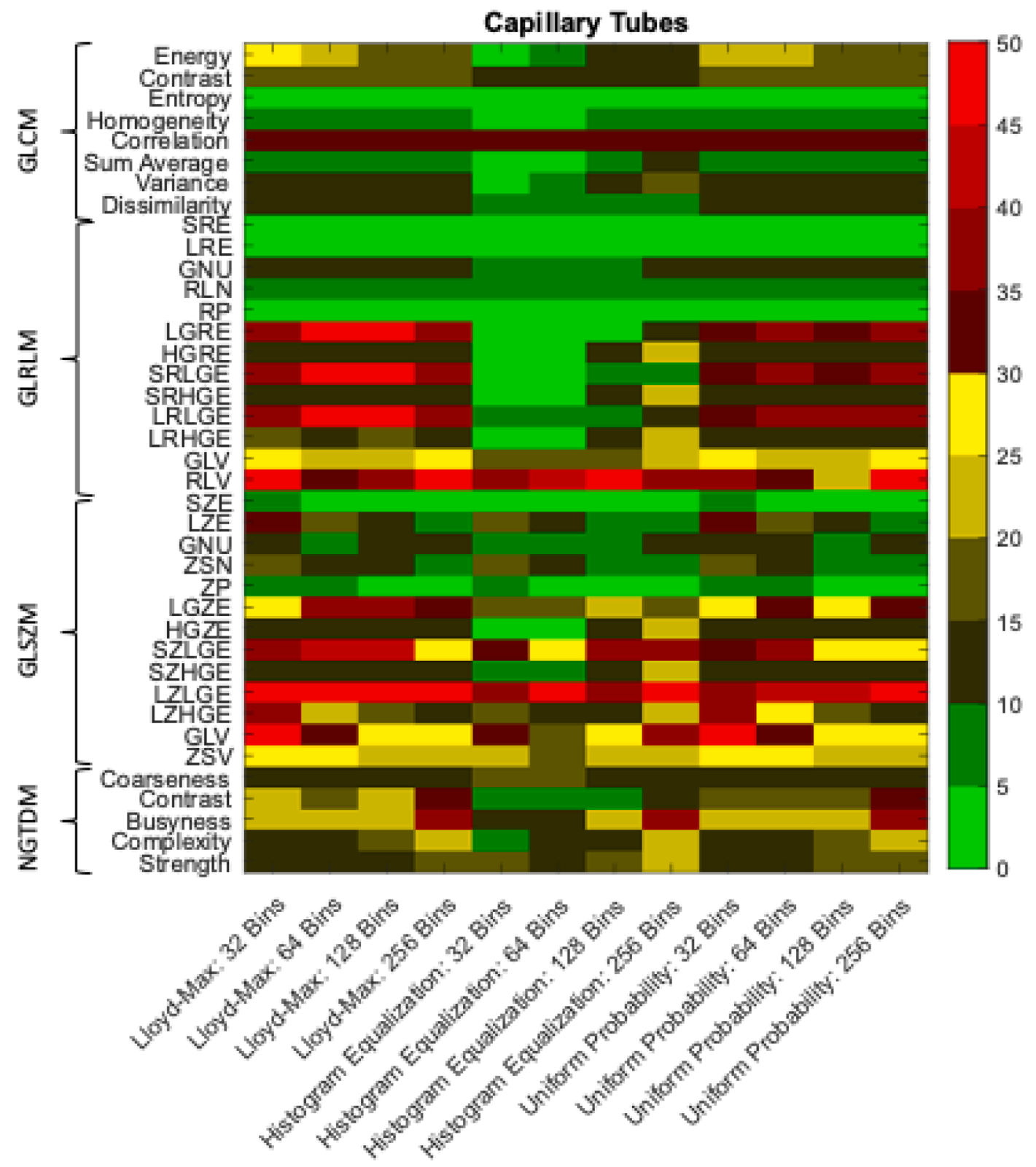

Fig. 6. CV\% for all texture features extracted from ROI_Cap. Histogram equalization using 32 bin levels results in the most texture features with CV\% below $10 \%$ (light and dark green) followed closely by histogram equalization using 64 intensity levels. A total of 7 texture features maintain a CV\% under 10\%. (For interpretation of the references to colour in this figure legend, the reader is referred to the web version of this article.)

Equalization results in higher contrast than the original image, and the other two methods result in similar contrast after quantization. While construction of and inclusion of a texture phantom gives investigators higher control over the types of image heterogeneity for extraction of texture features, there is no guarantee that these synthetic textures can replicate tumor heterogeneity. Without certainty that the underlying phantom structures for texture analysis are providing a useful surrogate for analysis, it is important to verify that the phantom-based texture features relate to those found clinically. The high values of $|\mathrm{SCC}|$ indicate that the heterogeneity within the phantoms, measured using the texture features, is highly correlated with real anatomy and that the synthetic phantom was an appropriate surrogate for the CV\%. The preprocessing options under consideration for this work appeared not to impact the repeatability of the texture features. There are two shortcomings of this work could result in seeing an impact on the repeatability of features. The first shortcoming is the sample size for test-retest, consideration of all liver and pancreas patients (left kidney always included in the image) resulted in 165 set up images with only 18 meeting criteria for inclusion. Larger sample size could produce more concreate CCCs even though Lin's CCC is valid for as few as 10 samples. The second shortcoming is the use of rigidly transferring contours. While rigidly transferring contours from one image series to the second eliminates contouring variability between the images and eliminates volume effects, changes in anatomy may lead to inclusion or exclusion of tissues of interest.

The strength of texture features is the ability to quantify underlying patterns imperceptible to the human eye. The known physical construction and appearance of the texture inserts permits us to hypothesize how some texture features may behave across the four texture inserts. It is reasonable to expect certain texture features to have higher or lower values due to a given phantom's structure when compared to the same feature extracted from the other inserts. Feature variation across the inserts can be seen using the first image acquired of the texture phantom and calculated using histogram equalization and 64 intensity levels. For 

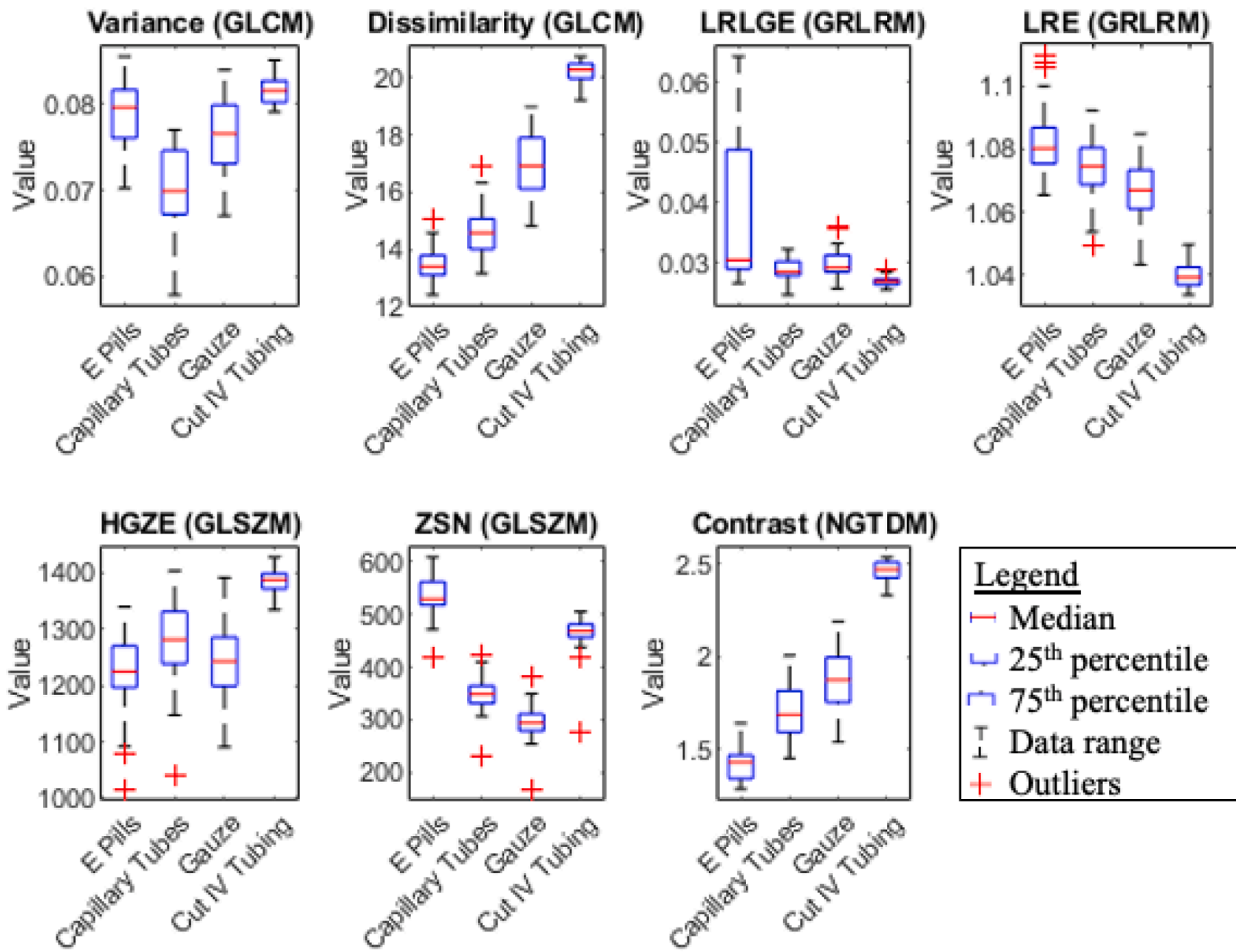

Fig. 7. displays a sample of features with a $\mathrm{CV} \%<10 \%$ extracted from ROIs processed using histogram equalization that were quantized to $64 \mathrm{~Gy}$ level intensity levels. All plotted features had at least one group of statistically different ( $p$-value $<0.01$ ) feature values. The $y$-axis is the value for the texture feature and the $\mathrm{x}$-axis labels are the ROI types in the phantom the features were calculated from. The box plots indicate that some features are sensitive across all texture inserts while others are impacted to a larger degree by only one texture insert.

Table 3

Texture features extracted from the GTV with a CCC $>0.80$. The left column contains the texture features along with their class. A checkmark indicates the CCC $>0.80$ and $\times$ indicates a CCC $<0.80$. The bottom row is the tally for features meeting or exceeding a CCC $=0.80$ for each quantization method and number of bin levels. The final column indicates whether a feature's CCC consistently high across all method combinations.

\begin{tabular}{|c|c|c|c|c|c|c|c|c|c|c|c|c|c|c|}
\hline \multirow[t]{2}{*}{ Feature Class: texture features } & \multirow{2}{*}{$\frac{\text { Algorithm }}{\text { Levels }}$} & \multicolumn{4}{|c|}{ Histogram Equalization } & \multicolumn{4}{|c|}{ Uniform Probability } & \multicolumn{4}{|c|}{ Lloyd-Max } & \multirow[t]{2}{*}{ Consistant across all methods: } \\
\hline & & 32 & 64 & 128 & 256 & 32 & 64 & 128 & 256 & 32 & 64 & 128 & 256 & \\
\hline GLRLM: GLN & & $\checkmark$ & $\checkmark$ & $\checkmark$ & $\checkmark$ & $\checkmark$ & $\checkmark$ & $\checkmark$ & $\checkmark$ & $\checkmark$ & $\checkmark$ & $\checkmark$ & $\checkmark$ & Yes \\
\hline GLRLM: RLN & & $\checkmark$ & $\checkmark$ & $\checkmark$ & $\checkmark$ & $\checkmark$ & $\checkmark$ & $\checkmark$ & $\checkmark$ & $\checkmark$ & $\checkmark$ & $\checkmark$ & $\checkmark$ & Yes \\
\hline GLSZM: GLN & & $\checkmark$ & $\checkmark$ & $\checkmark$ & $\checkmark$ & $\checkmark$ & $\checkmark$ & $\checkmark$ & $\checkmark$ & $\checkmark$ & $\checkmark$ & $\checkmark$ & $\checkmark$ & Yes \\
\hline GLSZM: ZSN & & $\checkmark$ & $\checkmark$ & $\checkmark$ & $\checkmark$ & $\checkmark$ & $\checkmark$ & $\checkmark$ & $\checkmark$ & $\checkmark$ & $\checkmark$ & $\checkmark$ & $\checkmark$ & Yes \\
\hline GLSZM: ZSV & & $\checkmark$ & $\checkmark$ & $\checkmark$ & $\checkmark$ & $\checkmark$ & $\checkmark$ & $\checkmark$ & $\checkmark$ & $\checkmark$ & $\checkmark$ & $\checkmark$ & $\checkmark$ & Yes \\
\hline NGTDM: Coarseness & & $\checkmark$ & $\checkmark$ & $\checkmark$ & $\checkmark$ & $\checkmark$ & $\checkmark$ & $\checkmark$ & $\checkmark$ & $\checkmark$ & $\checkmark$ & $\checkmark$ & $\checkmark$ & Yes \\
\hline NGTDM: Busyness & & $\checkmark$ & $\checkmark$ & $\checkmark$ & $\checkmark$ & $\checkmark$ & $\checkmark$ & $\checkmark$ & $\checkmark$ & $\checkmark$ & $\checkmark$ & $\checkmark$ & $\checkmark$ & Yes \\
\hline NGTDM: Strength & & $\checkmark$ & $\checkmark$ & $\checkmark$ & $\checkmark$ & $\checkmark$ & $\checkmark$ & $\checkmark$ & $\checkmark$ & $\checkmark$ & $\checkmark$ & $\checkmark$ & $\checkmark$ & Yes \\
\hline GLSZM: GLV & & $\checkmark$ & $\checkmark$ & $\checkmark$ & $\checkmark$ & $\mathrm{x}$ & $\checkmark$ & $\checkmark$ & $\checkmark$ & $\checkmark$ & $\checkmark$ & $\checkmark$ & $\checkmark$ & No \\
\hline GLCM: Energy & & $\mathrm{x}$ & $\mathrm{x}$ & $\mathrm{x}$ & $\mathrm{x}$ & $\checkmark$ & $\checkmark$ & $\checkmark$ & $\mathrm{x}$ & $\mathrm{x}$ & $\checkmark$ & $\mathrm{x}$ & $\mathrm{x}$ & No \\
\hline GLCM: Entropy & & $\mathrm{x}$ & $\mathrm{x}$ & $\mathrm{x}$ & $\mathrm{x}$ & $\checkmark$ & $\checkmark$ & $\mathrm{x}$ & $\mathrm{x}$ & $\mathrm{x}$ & $\checkmark$ & $\mathrm{x}$ & $\mathrm{x}$ & No \\
\hline NGTDM: Contrast & & $\mathrm{x}$ & $\mathrm{x}$ & $\checkmark$ & $\checkmark$ & $\mathrm{x}$ & $\mathrm{x}$ & $\mathrm{x}$ & $\checkmark$ & $\mathrm{x}$ & $\mathrm{x}$ & $\mathrm{x}$ & $\mathrm{x}$ & No \\
\hline GLSZM: LZHGE & & $\mathrm{x}$ & $\mathrm{x}$ & $\mathrm{x}$ & $\mathrm{x}$ & $\checkmark$ & $\mathrm{x}$ & $\mathrm{x}$ & $\mathrm{x}$ & $\checkmark$ & $\mathrm{x}$ & $\mathrm{x}$ & $\mathrm{x}$ & No \\
\hline Combination Totals: & & 9 & 9 & 10 & 10 & 11 & 12 & 10 & 10 & 10 & 11 & 9 & 9 & \\
\hline
\end{tabular}

example, GLCM-based 'contrast' emphasizes the co-occurrence of voxels with large gray level intensity differences highly. The ROI_IV has many regions of high and low intensities bordering each other, and it is reasonable to expect a higher measure of contrast than the other ROIs.
This supposition is validated by the ROI_IV having the highest value. The next highest value, normalized by the value of the ROI_IV, is from ROI_G (0.77), then ROI_Cap (0.51), and the lowest value was calculated from ROIE (0.48). The ANOVA analysis confirms that GLCM 'contrast' is 
Table 4

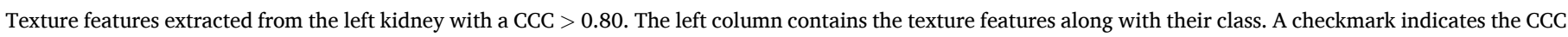

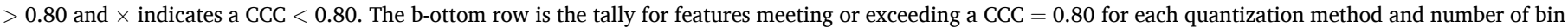
levels. The final column indicates whether a feature's CCC consistently high across all method combinations.

\begin{tabular}{|c|c|c|c|c|c|c|c|c|c|c|c|c|c|c|}
\hline \multirow[t]{2}{*}{ Feature Class: texture features } & \multirow{2}{*}{$\frac{\text { Algorithm }}{\text { Levels }}$} & \multicolumn{4}{|c|}{ Histogram Equalization } & \multicolumn{4}{|c|}{ Uniform Probability } & \multicolumn{4}{|c|}{ Lloyd-Max } & \multirow[t]{2}{*}{ Consistant across all methods: } \\
\hline & & 32 & 64 & 128 & 256 & 32 & 64 & 128 & 256 & 32 & 64 & 128 & 256 & \\
\hline GLRLM: GLN & & $\checkmark$ & $\checkmark$ & $\checkmark$ & $\checkmark$ & $\checkmark$ & $\checkmark$ & $\checkmark$ & $\checkmark$ & $\checkmark$ & $\checkmark$ & $\checkmark$ & $\checkmark$ & Yes \\
\hline GLRLM: RLN & & $\checkmark$ & $\checkmark$ & $\checkmark$ & $\checkmark$ & $\checkmark$ & $\checkmark$ & $\checkmark$ & $\checkmark$ & $\checkmark$ & $\checkmark$ & $\checkmark$ & $\checkmark$ & Yes \\
\hline GLSZM: GLN & & $\checkmark$ & $\checkmark$ & $\checkmark$ & $\checkmark$ & $\checkmark$ & $\checkmark$ & $\checkmark$ & $\checkmark$ & $\checkmark$ & $\checkmark$ & $\checkmark$ & $\checkmark$ & Yes \\
\hline NGTDM: Coarseness & & $\checkmark$ & $\checkmark$ & $\checkmark$ & $\checkmark$ & $\checkmark$ & $\checkmark$ & $\checkmark$ & $\checkmark$ & $\checkmark$ & $\checkmark$ & $\checkmark$ & $\checkmark$ & Yes \\
\hline NGTDM: Busyness & & $\checkmark$ & $\checkmark$ & $\checkmark$ & $\checkmark$ & $\checkmark$ & $\checkmark$ & $\checkmark$ & $\checkmark$ & $\checkmark$ & $\checkmark$ & $\checkmark$ & $\checkmark$ & Yes \\
\hline GLSZM: ZSN & & $\checkmark$ & $\checkmark$ & $\checkmark$ & $\checkmark$ & $\mathrm{x}$ & $\checkmark$ & $\checkmark$ & $\checkmark$ & $\checkmark$ & $\checkmark$ & $\checkmark$ & $\checkmark$ & No \\
\hline NGTDM: Strength & & $\checkmark$ & $\mathrm{x}$ & $\mathrm{x}$ & $\mathrm{x}$ & $\checkmark$ & $\checkmark$ & $\checkmark$ & $\checkmark$ & $\checkmark$ & $\checkmark$ & $\checkmark$ & $\checkmark$ & No \\
\hline GLSZM: ZSV & & $\checkmark$ & $\mathrm{x}$ & $\mathrm{x}$ & $\mathrm{x}$ & $\checkmark$ & $\mathrm{x}$ & $\mathrm{x}$ & $\checkmark$ & $\checkmark$ & $\mathrm{x}$ & $\mathrm{x}$ & $\checkmark$ & No \\
\hline GLSZM: LZLGE & & $\mathrm{x}$ & $\mathrm{x}$ & $\mathrm{x}$ & $\mathrm{x}$ & $\checkmark$ & $\mathrm{x}$ & $\mathrm{x}$ & $\mathrm{x}$ & $\checkmark$ & $\checkmark$ & $\mathrm{x}$ & $\mathrm{x}$ & No \\
\hline GLSZM: GLV & & $\checkmark$ & $\mathrm{x}$ & $\mathrm{x}$ & $\mathrm{x}$ & $\mathrm{x}$ & $\mathrm{x}$ & $\mathrm{x}$ & $\checkmark$ & $\mathrm{x}$ & $\mathrm{x}$ & $\mathrm{x}$ & $\mathrm{x}$ & No \\
\hline GLSZM: LZE & & $\mathrm{x}$ & $\mathrm{x}$ & $\mathrm{x}$ & $\mathrm{x}$ & $\checkmark$ & $\mathrm{x}$ & $\mathrm{x}$ & $\mathrm{x}$ & $\checkmark$ & $\mathrm{x}$ & $\mathrm{x}$ & $\mathrm{x}$ & No \\
\hline GLSZM: LGZE & & $\mathrm{x}$ & $\checkmark$ & $\mathrm{x}$ & $\mathrm{x}$ & $\mathrm{x}$ & $\mathrm{x}$ & $\mathrm{x}$ & $\mathrm{x}$ & $\mathrm{x}$ & $\mathrm{x}$ & $\mathrm{x}$ & $\mathrm{x}$ & No \\
\hline GLSZM: LZHGE & & $\mathrm{x}$ & $\mathrm{x}$ & $\checkmark$ & $\mathrm{x}$ & $\mathrm{x}$ & $\mathrm{x}$ & $\mathrm{x}$ & $\mathrm{x}$ & $\mathrm{x}$ & $\mathrm{x}$ & $\mathrm{x}$ & $\mathrm{x}$ & No \\
\hline GLSZM: SZLGE & & $\mathrm{x}$ & $\checkmark$ & $\mathrm{x}$ & $\mathrm{x}$ & $\mathrm{x}$ & $\mathrm{x}$ & $\mathrm{x}$ & $\mathrm{x}$ & $\mathrm{x}$ & $\mathrm{x}$ & $\mathrm{x}$ & $\mathrm{x}$ & No \\
\hline Combination Totals: & & 9 & 8 & 7 & 6 & 9 & 7 & 7 & 9 & 10 & 8 & 7 & 8 & \\
\hline
\end{tabular}

sensitive to the ROI type.

Although the low field strength images have lower signal-to-noise ratios (SNR) than diagnostic images the clinical bSSFP pulse sequence delivers the highest possible SNR. The longitudinal datasets produced during routine treatments provide a tantalizing opportunity for texture analysis. The limited number of pulse sequences available for clinical set up images is an advantage for texture analysis. Limiting image acquisition parameters provide stability to acquisition parameters across all patients resulting in a more extensive image database. By taking advantage of the similar images, the repeatability of clinical $0.35 \mathrm{~T}$ images acquired with bSSFP was investigated. The impact of the quantization algorithm and the number of intensity levels on repeatability is less apparent. There were not any combinations that produced superior repeatability. However, there were two sets of GLRLM and GLSZM features selected that measure similar qualities. GLSZM GLN is an updated GLRLM GLN where the measurement is based upon zones instead of run lengths. Similarly, GLSZM ZSN is an updated GLRLM RLN with the texture feature measuring zones instead of run lengths. While it is likely that GLRLM and GLSZM-based features are redundant, a texture feature from either class could contain better prognostic ability.

Currently, there are no clinical repeatability studies using the low field strength MR set up images acquired with the bSSFP pulse sequence. However, a previous study using a bSSFP pulse sequence by Mahon et al. measured the repeatability of texture features in $1.5 \mathrm{~T}$ MR images using CCC. Their work established $64.4 \%$ of features extracted from tumors were repeatable (CCC $\geq 0.9$ ). Additionally, $67.8 \%$ of the features extracted from normal muscle tissue were repeatable as well [32]. Image noise is known to reduce the repeatability of texture features in MR images, and the increased noise in our low field strength images is reflected in the results of having fewer texture features meeting our threshold of CCC $\geq 0.8$. A low threshold was selected for this study for two reasons. Previous test-retest studies of radiomics texture features have used a CCC $=0.85$ to remove features in a prescreening step $[31,67]$. However, the purpose of the test-retest for this work is not to eliminate features, just to explore the impact of each quantization method along with the number of bins on repeatability. All texture features that were repeatable in the left kidney were also repeatable in the GTV. The differences in the numbers of repeatable features between the GTV ( 8 across all combinations) and the left kidney ( 5 features) are likely due to positional differences between acquisitions, as seen in the coronal view (right column) of Fig. 2.

Volume dependence has been shown to artificially inflate the repeatability of some texture features [68]. Cross-referencing the repeatable texture features with volume dependence was performed to indicate features that could be substantially influenced by volume dependence. A texture feature with high volume dependence could be repeatable due to the identical volumes in the test-retest image pair. Highly volume dependent features could be encoding ROI volume instead of tumor heterogeneity and result in redundant volumetric information. However, steps can be taken to minimize volume dependence. A popular choice to avoid the hidden volume effect is to perform a correlation study and remove texture features that are highly correlated with volume from the remainder of the study. A second option to explore is the mitigation of the impact of volume by further development of texture features that reduce the impact of volume during calculation. Fave et al successfully modified five texture feature definitions by including different voxel counts in their definitions, resulting in lower volume correlation [69].

Several popular texture features extracted from low field strength MR images were demonstrated as predictive of clinical complete response in rectal cancer patients [27]. The study was performed using an agnostic approach with no pre-screening of features extracted from low field strength images and a small patient cohort. A pilot texture analysis study published by our group using low field strength MR images of pancreatic cancer patients showed promising predictive performance for response [70]. Texture features with the highest predictive ability were calculated using the Histogram Equalization method and 64 Gy-level intensity levels. Interest in texture features is based on their ability to perform in a clinical setting. The relative ease of extracting texture features has led to the evaluation of their predictive, prognostic, and classification abilities without baseline benchmarking feature behavior in response to differences in important preprocessing steps. Although the method to quantize the $1.5 \mathrm{~T}$ T2-weighted and apparent diffusing coefficient map images was not disclosed, Abdollahi et al. used texture features calculated using $64 \mathrm{~Gy}$-level intensity values to predict response in prostate cancer patients and to predict Gleason score [71].

\section{Conclusion}

Low field strength MR images have been brought into the patient care path to serve as image guidance in RT treatments. Although generally of lower quality than diagnostic MR images, they still produce far better contrast than x-ray based set up modalities. Texture analysis using these images could provide valuable prognostic and predictive information about the disease during treatment. This work investigated the impact of three quantization methods and the number of intensity levels on texture feature variability, verified the most stable calculation method could maintain sensitivity to the phantom texture inserts, and 
investigated the impact on repeatability in clinical set up images. Histogram equalization with 64 intensity levels resulted in the least variability in texture features extracted from the four different phantom texture inserts. No combination of quantization algorithm and the number of intensity levels were superior in the test-retest portion of the study using the GTV and left kidney. However, eight texture features displayed fair repeatability in the GTV. Fewer features extracted from the kidney were repeatable, this was likely due to positional changes. The large number of stable and repeatable features extracted from the low field strength MR images supports the use of texture features for building predictive and prognostic models. The method used for ROI preprocessing in texture analysis studies using low field strength MR images for building models should always be considered to obtain reliable results.

\section{Declaration of Competing Interest}

The authors declare that they have no known competing financial interests or personal relationships that could have appeared to influence the work reported in this paper.

\section{References}

[1] Gillies RJ, Kinahan PE, Hricak H. Radiomics: images are more than pictures, they are data. Radiology 2016;278:563-77.

[2] Castellano G, Bonilha L, Li LM, Cendes F. Texture analysis of medical images. Clin Radiol 2004;59:1061-9.

[3] Kumar V, Gu YH, Basu S, Berglund A, Eschrich SA, Schabath MB, et al. Radiomics: the process and the challenges. Magn Reson Imaging 2012;30:1234-48.

[4] Avanzo M, Stancanello J, El Naqa I. Beyond imaging: the promise of radiomics. Physica Med 2017:38:122-39.

[5] Lambin P, Rios-Velazquez E, Leijenaar R, Carvalho S, van Stiphout R, Granton P, et al. Radiomics: extracting more information from medical images using advanced feature analysis. Eur J Cancer 2012;48:441-6.

[6] Rutman AM, Kuo MD. Radiogenomics: creating a link between molecular diagnostics and diagnostic imaging. Eur J Radiol 2009;70:232-41.

[7] Tixier F, Le Rest CC, Hatt M, Albarghach N, Pradier O, Metges JP, et al. Intratumor Heterogeneity characterized by textural features on baseline F-18-FDG PET images predicts response to concomitant radiochemotherapy in esophageal cancer. J Nucl Med 2011;52:369-78.

[8] Sala E, Mema E, Himoto Y, Veeraraghavan H, Brenton JD, Snyder A, et al. Unravelling tumour heterogeneity using next-generation imaging: radiomics, radiogenomics, and habitat imaging. Clin Radiol 2017;72:3-10.

[9] Jin KT, He KF, Teng F, Han N, Li GL, Xu ZZ, et al. Heterogeneity in primary tumors and corresponding metastases: could it provide us with any hints to personalize cancer therapy? Personalized Med 2011;8:175-82.

[10] Almendro V, Marusyk A, Polyak K. Cellular heterogeneity and molecular evolution in cancer. In: Abbas AK, Galli SJ, Howley PM, editors. Annual review of pathology: mechanisms of disease, vol. 8. Palo Alto: Annual Reviews; 2013. p. 277-302.

[11] Burrell RA, McGranahan N, Bartek J, Swanton C. The causes and consequences of genetic heterogeneity in cancer evolution. Nature 2013;501:338.

[12] Tu CF, Oi P, Li XY, Mo YZ, Li XL, Xiong W, et al. Tumor heterogeneity: the challenge of precision medicine. Prog Biochem Biophys. 2015;42:881-90.

[13] Yang F, Ford JC, Dogan N, Padgett KR, Breto AL, Abramowitz MC, et al. Magnetic resonance imaging (MRI)-based radiomics for prostate cancer radiotherapy. Transl Androl Urol 2018;7:445-58.

[14] Yang F, Thomas MA, Dehdashti F, Grigsby PW. Temporal analysis of intratumoral metabolic heterogeneity characterized by textural features in cervical cancer. Eur J Nucl Med Mol Imaging 2013;40:716-27.

[15] Hanania AN, Bantis LE, Feng Z, Wang H, Tamm EP, Katz MH, et al. Quantitative imaging to evaluate malignant potential of IPMNs. Oncotarget 2016;7:85776-84.

[16] Permuth JB, Choi J, Balarunathan Y, Kim J, Chen D-T, Chen L, et al. Combining radiomic features with a miRNA classifier may improve prediction of malignant pathology for pancreatic intraductal papillary mucinous neoplasms. Oncotarget 2016;7:85785-97.

[17] Fan M, Li H, Wang S, Zheng B, Zhang J, Li L. Radiomic analysis reveals DCE-MRI features for prediction of molecular subtypes of breast cancer. PLoS ONE 2017;12: e0171683.

[18] Cunliffe A, Armato 3rd SG, Castillo R, Pham N, Guerrero T, Al-Hallaq HA. Lung texture in serial thoracic computed tomography scans: correlation of radiomicsbased features with radiation therapy dose and radiation pneumonitis development. Int J Radiat Oncol Biol Phys 2015;91:1048-56.

[19] Abdollahi H, Tanha K, Mofid B, Razzaghdoust A, Saadipoor A, Khalafi L, et al. MRI radiomic analysis of IMRT-induced bladder wall changes in prostate cancer patients: a relationship with radiation dose and toxicity. J Med Imag Rad Sci 2019; 50:252-60.

[20] Scalco E, Fiorino C, Cattaneo GM, Sanguineti G, Rizzo G. Texture analysis for the assessment of structural changes in parotid glands induced by radiotherapy. Radiother Oncol 2013;109:384-7.
[21] Yang D, Rao G, Martinez J, Veeraraghavan A, Rao A. Evaluation of tumor-derived MRI-texture features for discrimination of molecular subtypes and prediction of 12month survival status in glioblastoma. Med Phys 2015;42:6725-35.

[22] Coroller TP, Agrawal V, Narayan V, Hou Y, Grossmann P, Lee SW, et al. Radiomic phenotype features predict pathological response in non-small cell lung cancer. Radiother Oncol. 2016;119:480-6.

[23] Liu J, Mao Y, Li Z, Zhang D, Zhang Z, Hao S, et al. Use of texture analysis based on contrast-enhanced MRI to predict treatment response to chemoradiotherapy in nasopharyngeal carcinoma. J Magn Reson Imaging 2016;44:445-55.

[24] van Dijk LV, Brouwer CL, van der Schaaf A, Burgerhof JGM, Beukinga RJ, Langendijk JA, et al. CT image biomarkers to improve patient-specific prediction of radiation-induced xerostomia and sticky saliva. Radiother Oncol 2017;122: 185-91.

[25] Yang F, Young L, Grigsby P. Predictive value of standardized intratumoral metabolic heterogeneity in locally advanced cervical cancer treated with chemoradiation. Int J Gynecol Cancer 2016;26:777-84.

[26] Mutic S, Dempsey JF. The ViewRay system: magnetic resonance-guided and controlled radiotherapy. Semin Rad Oncol 2014;24:196-9.

[27] Boldrini L, Cusumano D, Chiloiro G, Casà C, Masciocchi C, Lenkowicz J, et al. Delta radiomics for rectal cancer response prediction with hybrid $0.35 \mathrm{~T}$ magnetic resonance-guided radiotherapy (MRgRT): a hypothesis-generating study for an innovative personalized medicine approach. Radiol Med (Torino) 2018.

[28] Yip SSF, Aerts H. Applications and limitations of radiomics. Phys Med Biol 2016; 61:R150-66.

[29] Yang F, Simpson G, Young L, Ford J, Dogan N, Wang L. Impact of contouring variability on oncological PET radiomics features in the lung. Scientific Rep 2020. p. 369.

[30] Nyflot MJ, Yang F, Byrd D, Bowen SR, Sandison GA, Kinahan PE. Quantitative radiomics: impact of stochastic effects on textural feature analysis implies the need for standards. J Med Imaging 2015;2:13.

[31] Peerlings J, Woodruff HC, Winfield JM, Ibrahim A, Van Beers BE, Heerschap A, et al. Stability of radiomics features in apparent diffusion coefficient maps from a multi-centre test-retest trial. Sci Rep 2019;9:10.

[32] Mahon RN, Hugo GD, Weiss E. Repeatability of texture features derived from magnetic resonance and computed tomography imaging and use in predictive models for non-small cell lung cancer outcome. Phys Med Biol 2019.

[33] Waugh SA, Lerski RA, Bidaut L, Thompson AM. The influence of field strength and different clinical breast MRI protocols on the outcome of texture analysis using foam phantoms. Med Phys 2011;38:5058-66.

[34] Fiset S, Welch ML, Weiss J, Pintilie M, Conway JL, Milosevic M, et al. Repeatability and reproducibility of MRI-based radiomic features in cervical cancer. Radiother Oncol 2019;135:107-14.

[35] Schwier M, van Griethuysen J, Vangel MG, Pieper S, Peled S, Tempany C, et al. Repeatability of multiparametric prostate MRI radiomics features. Sci Rep 2019;9: 9441.

[36] Mayerhoefer ME, Szomolanyi P, Jirak D, Materka A, Trattnig S. Effects of MRI acquisition parameter variations and protocol heterogeneity on the results of texture analysis and pattern discrimination: an application-oriented study. Med Phys 2009;36:1236-43.

[37] Ford J, Dogan N, Young L, Yang F. Quantitative radiomics: impact of pulse sequence parameter selection on MRI-based textural features of the brain. Contrast Media Mol Imaging. 2018;2018:1729071.

[38] Yang F, Dogan N, Stoyanova R, Ford JC. Evaluation of radiomic texture feature error due to MRI acquisition and reconstruction: a simulation study utilizing ground truth. Physica Medica 2018;50:26-36.

[39] Herlidou-Meme S, Constans JM, Carsin B, Olivie D, Eliat PA, Nadal-Desbarats L, et al. MRI texture analysis on texture test objects, normal brain and intracranial tumors. Magn Reson Imaging 2003;21:989-93.

[40] Jirak D, Dezortova M, Hajek M. Phantoms for texture analysis of MR images. Longterm and multi-center study. Med Phys 2004;31:616-22.

[41] Savio SJ, Harrison LCV, Luukkaala T, Heinonen T, Dastidar P, Soimakallio S, et al. Effect of slice thickness on brain magnetic resonance image texture analysis. Biomed Eng Online 2010;9:14.

[42] Nyúl LG, Udupa JK. On standardizing the MR image intensity scale. Magn Reson Med 1999;42:1072-81.

[43] Collewet G, Strzelecki M, Mariette F. Influence of MRI acquisition protocols and image intensity normalization methods on texture classification. Magn Reson Imaging 2004;22:81-91.

[44] Klüter S. Technical design and concept of a 0.35 T MR-Linac. Clinical and translational. Rad Oncol 2019;18:98-101.

[45] Traverso A, Kazmierski M, Welch ML, Weiss J, Fiset S, Foltz WD, et al. Sensitivity of radiomic features to inter-observer variability and image pre-processing in Apparent Diffusion Coefficient (ADC) maps of cervix cancer patients. Radiother Oncol 2019.

[46] Brynolfsson P, Nilsson D, Torheim T, Asklund T, Karlsson CT, Trygg J, et al. Haralick texture features from apparent diffusion coefficient (ADC) MRI images depend on imaging and pre-processing parameters. Sci Rep 2017;7:4041.

[47] Lloyd S. Least squares quantization in PCM. IEEE Trans Inf Theory 1982;28: 129-37.

[48] Max J. Quantizing for minimum distortion. IRE Trans Inf Theory 1960;6:7-12.

[49] Vallières M. A radiomics model from joint FDG-PET and MRI texture features for the prediction of lun metastases in soft-tissue sarcomas of the extremities. Phys Med Biol 2015;60:5471-96.

[50] Haralick RM, Shanmugam K, Dinstein I. Textural features for image classification. IEEE Trans Syst Man Cybern 1973;SMC3:610-21. 
[51] Haralick RM. Statistical and structural approaches to texture. Proc IEEE 1979;67: 786-804.

[52] Assefa D, Keller H, Menard C, Laperriere N, Ferrari RJ, Yeung I. Robust texture features for response monitoring of glioblastoma multiforme on T1-weighted and T2-FLAIR MR images: a preliminary investigation in terms of identification and segmentation. Med Phys 2010;37:1722-36.

[53] Amadasun M, King R. Textural features corresponding to textural properties. IEEE Trans Syst Man Cybern 1989;19:1264-74.

[54] Galloway MM. Texture analysis using gray level run lengths. Comput Graph Image Process 1975;4:172-9.

[55] Chu A, Sehgal CM, Greenleaf JF. Use of gray value distribution of run lengths for texture analysis. Pattern Recogn Lett 1990;11:415-9.

[56] Dasarathy BV, Holder EB. Image characterizations based on joint gray level—run length distributions. Pattern Recogn Lett 1991;12:497-502.

[57] Thibault G, Fertil B, Navarro C, Pereira S, Lévy N, Sequeira J, et al. Texture indexes and gray level size zone matrix application to cell nuclei classification 2009.

[58] Zwanenburg ALS, Vallières M, Löck S. Image biomarker standardization initiative. 2019:arXiv preprint arXiv:1612.07003.

[59] Yan J, Chu-Shern JL, Loi HY. Impact of image reconstruction settings on texture features in 18F-FDG PET. J Nucl Med 2015;56:1667.

[60] Fisher RA. Studies in crop variation. I. An examination of the yield of dressed grain from Broadbalk. J Agric Sci 1921;11:107-35.

[61] Lin LI. A concordance correlation-coefficient to evaluate reproducibility. Biometrics 1989;45:255-68.

[62] McBride GB. A proposal for strength-of-agreement criteria for Lin's concordance correlation coefficient. National Institute of Water \& Atmospheric Research Ltd. 2005:1-10.
[63] Balagurunathan Y, Gu Y, Wang H. Reproducibility and prognosis of quantitative features extracted from CT images. Transl Oncol 2014;7:72.

[64] Balagurunathan Y, Kumar V, Gu Y, Kim J, Wang H, Liu Y, et al. Test-retest reproducibility analysis of lung CT image features. J Digit Imaging 2014;27: 805-23.

[65] van Timmeren JE, Leijenaar RTH, van Elmpt W, Wang JZ, Zhang Z, Dekker A, et al. Test-retest data for radiomics feature stability analysis: generalizable or studyspecific? Tomography 2016;2:361-5.

[66] Garpebring A, Brynolfsson P, Kuess P, Georg D, Helbich TH, Nyholm T, et al. Density estimation of grey-level co-occurrence matrices for image texture analysis. Phys Med Biol 2018;63:195017.

[67] Larue RTHM, Van De Voorde L, van Timmeren JE, Leijenaar RTH, Berbée M, Sosef MN, et al. 4DCT imaging to assess radiomics feature stability: an investigation for thoracic cancers. Radiother Oncol 2017;125:147-53.

[68] Fave X, Mackin D, Yang J, Zhang J, Fried D, Balter P, et al. Can radiomics features be reproducibly measured from CBCT images for patients with non-small cell lung cancer? Med Phys 2015;42:6784-97.

[69] Fave X, Zhang L, Yang J. Impact of image preprocessing on the volume dependence and prognostic potential of radiomics features in non-small cell lung cancer. Transl Cancer Res 2016;5:349.

[70] Simpson G, Spieler B, Dogan N, Portelance L, Mellon EA, Kwon D, et al. Predictive value of $0.35 \mathrm{~T}$ magnetic resonance imaging radiomic features in stereotactic ablative body radiotherapy of pancreatic cancer: a pilot study. Med Phys 2020.

[71] Abdollahi H, Moid B, Shiri I, Razzaghdoust A, Saadipoor A, Mahdavi A, et al. Machine learning-based radiomic models to predict intensity-modulated radiation therapy response, Gleason score and stage in prostate cancer. Radiol Med (Torino) $2019 ; 124$. 